\title{
American option valuation under time changed tempered stable Lévy processes
}

\author{
Xiaoli Gong* Xintian Zhuang \\ School of Business Administration, Northeastern University, Shenyang, 110169, China
}

\begin{abstract}
Given that the underlying assets in financial markets exhibit stylized facts such as leptokurtosis, asymmetry, clustering properties and heteroskedasticity effect, this paper presents a novel model for pricing American option under the assumptions that the stock prices processes are govern by time changed tempered stable Lévy process. As this model is constructed by introducing random time changes into tempered stable (TS) processes which specially refer to normal tempered stable (NTS) distribution as well as classical tempered stable (CTS) distribution, it permits infinite jumps as well as capturing random varying time in stochastic volatility, consequently taking into account the empirical facts such as leptokurtosis, skewness and volatility clustering behaviors. We employ the Fourier-cosine technique to calculate American option and propose the improved Particle Swarm optimization (IPSO) intelligent algorithm for model calibration. To demonstrate the advantage of the constructed model, we carry out empirical research on American index option in financial markets across wide ranges of models, with the time changing normal tempered stable distribution model yielding a superior performance than others.
\end{abstract}

Keywords: stylized facts; tempered stable processes; Fourier-cosine technique; improved Particle Swarm optimization

\section{Introduction}

In the seminal paper of Black-Scholes, asset prices are assumed to behave according to the geometric Brownian motion. While the traditional BS option pricing formula contradicts the fact that density functions of financial asset returns exhibit abnormality such as heavy tails and asymmetries in market data as well as volatility smile in option markets [1, 2]. Considerable empirical evidence has demonstrated that normal distribution assumption is not sufficiently flexible to explain the dynamics of derivative products [3]. Vast efforts have been made to relax its restrictive assumptions. It has been illustrated in large amount of literatures that the distributions of asset returns exhibit leptokurtosis and clustering characteristics which give rise to extensive alternative jump diffusion models to accommodate stochastic volatility in returns variance and jumps behaviors in asset price processes $[4,5]$.

One alternative way to capture the high peak and fat tail properties in financial markets is to employ the class of infinite activity tempered stable processes which belong to infinite activity Lévy processes and have been validated superior to common infinite activity Lévy processes such as the Variance Gamma (VG) process presented by Madan and Carr [6], Normal Inverse Gaussian (NIG) process presented by Barndorff Nielsen [7]. The Lévy processes are able to be divided into finite activity Lévy processes and infinite activity Lévy processes, here we only involve the latter. When combining with stochastic volatility components in European option valuation, the tempered stable processes outperform other models [8]. The infinite activity Lévy models are attractive since they can capture jump patterns including jumps strength and jump structure exhibited by stocks returns more effectively. The tempered stable processes proposed by Rosinski [9] consist of classical tempered stable distribution process, normal tempered stable distribution process in [10] and rapidly decreasing tempered stable distribution process in [11] and so on. The TS distributions are generally gained by making $\alpha$ stable law multiply their corresponding Lévy measures generated from moment generating functions. This type of distribution is equipped with asymmetry properties, higher kurtosis and fatter tails than the normal distribution, which can better capture the stylized facts observed in financial asset returns. Moreover, its infinite activity characteristic can better describe the infinite jumps phenomenon existed in the underlying process $[12,13]$.

Concerning the time varying characteristics of volatility in underlying returns and option pricing models, Barndorff Nielsen and Shephard [14] conducted research on stochastic volatility processes with non-Gaussian components. Huang and $\mathrm{Wu}[15]$ studied a series of option pricing approaches and demonstrated that financial processes combining jumps and stochastic diffusion components are best suitable. Carr [16] pointed out that introducing stochastic volatility into Lévy processes allowed reproducing realistic implied volatility behaviors across maturities without requiring the time variation of parameters. Due to the dependence of increments in stock price processes the volatility smiles persist forward, after adding jumps it allows to reproduce both skews and smiles in underlying asset processes. In order to regenerate implied volatility, Schoutens [17] presented the Lévy driven stochastic volatility model framework to describe asset price process which depicts time changed Lévy processes out of an integrated Cox-Ingersoll-Ross (CIR) square root stochastic clock and this framework include

* Corresponding author. Tel.: +862483679139.

E-mail address: qdgongxiaoli@126.com 
VG-CIR model and NIG-CIR model and so on. It can increase or decrease the uncertainty in economy by speeding up or slowing down the elapsed time rate. Zaevski [18] turned attention to the stochastic volatility model with classical tempered stable Lévy jumps to more reasonably exhibit the jumps behavior of returns. Our enhancement over the prevailing is the extension from time changed classical tempered stable distribution (CTSSV) to time changed normal tempered stable distribution (NTSSV) to re-construct the time changed tempered stable model (TSSV) framework.

There have been a variety of derivative applications for time varying Lévy processes in financial markets. Yamazaki [19] adopted VG-CIR and NIG-CIR model under time varying Lévy process framework to approximately price average options using Gram-Charlier expansion technique. Itkin [20] and Carr [21] applied time varying Lévy process to volatility derivatives. Bianchi [22] investigated the pricing performance of credit default swap spreads assuming stochastic non-Gaussian Lévy process dynamics for the default intensity model. Kim [23] derived the analytic solution for quanto option pricing under the NTS model. While compared with standard European option, studies of pricing other derivatives about time changing tempered stable processes seem to be lacking. However, in the following, we present the American option pricing framework under the time changing tempered stable Lévy processes.

Regarding the American option valuation technique, enormous approaches have been proposed for Lévy processes $[24,25]$. From all of them we choose the Fourier-cosine method for TSSV models since its powerful calculation capacity to early exercise property as long as the characteristic functions of underlying assets are readily available. Compared to other fast Fourier transform (FFT) based techniques, it exhibits more accurate and efficient capacities, which make option parameters calibration possible. During the calculation, extrapolation technique presented can be used to price such option from Bermudan option quickly and accurately. The repeated Richardson extrapolation techniques are employed to enhance the computational efficiency and accuracy of American option.

It is illustrated that parameters estimated directly from real option prices are preferable than that estimated from asset returns data. Notice that the NTSSV model calibration is a complex nonlinear program problem. Li [26] proposed the characteristic function based spectral estimation method in CTSSV models for option pricing. However, it calls for Monte Carlo simulation which may lead to errors accordingly. Li [27] used the unscented Kalman filter to jointly identify stochastic volatility and jumps in pricing time changed infinite activity derivative models, while it required the corresponding known proposal density. The optimization problems of model calibrations are pretty complex and traditional algorithms utilized for calibration may not guarantee global minimum results. Therefore, there are strong requirements for developing sophisticated approaches in calibrations. Kennedy [28] proposed particle swarm optimization (PSO) algorithm based on swarm intelligence stochastic optimization algorithm which used iterative optimization calculation to find a set of parameter values under certain constraints so that some indicators can reach the maximum or minimum. This algorithm possesses memory and has fast search speed. Krink [29] verified the advantages of intelligent optimization algorithms in financial models applications. Fastrich [30] exploited differential evolution algorithm to predict the German stock portfolio. However, PSO algorithm is easy to fall into local optimum, resulting in relative larger error results. For that reason, Yang [31] proposed multi-basin PSO algorithm aimed at increasing diversity of particles, then validated the effectiveness of intelligent optimization algorithms through parameters estimation of Lévy option pricing models. Upon this, this paper presents an improved dynamic inertia weight PSO algorithm to improve the calibration efficiency, and applies it to the newly constructed model for parameters calibration.

The outstanding contribution of our work to the previous is that we bring stochastic time change in returns volatility into the normal tempered stable process to construct time changed normal tempered stable model, which is compared to time changed classical tempered stable model and NIGCIR model. The advantage of this newly constructed model framework over the prevailing is the reflection of infinite activity jump behaviors in underlying assets and clustering effects in time changing volatility at the same time. In addition, the Fourier-cosine method is exploited for American option valuation. In order to improve the efficiency of model calibration, improved PSO algorithm is employed to our newly constructed model, solving the poor performance of nonlinear optimization problems when utilizing local search techniques and traditional PSO algorithm.

The organization of the paper is as follows. Section 2 builds the time changed tempered stable Lévy processes by subordinating the extensively used infinite activity tempered stable Lévy processes to the integrated CIR process. In Section 3 we employ the Fourier-cosine method for American option with early exercise features where a novel quadrature based method and the FFT technique are utilized. Section 4 presents the improved PSO intelligent algorithm for model calibration. Then, we investigate the pricing deviation on OEX American options so as to assess the influences of leptokurtosis, skewness and volatility clustering on option valuation in Section 5 and finally conclude the paper in Section 6.

\section{Time changed tempered stable Lévy processes}


The advantage of stochastic process to describe stochastic volatility over regime switching methods and time series methods is that it can be effectively applied in later calibration because of the availability of analytical characteristic functions [32]. In this paper, we introduce the stochastic time varying into the tempered stable process framework by letting the variance of classical tempered stable distribution and normal tempered stable distribution change with time, which method had been used in Carr [33] for time changed Lévy processes in European option pricing covering VG-CIR and NIG-CIR models. We extend its framework via incorporating normal tempered stable distribution with random time change to capture the information content of markets and implement it in American option valuation.

Let $\varphi$ represent the characteristic function of tempered stable Lévy processes under risk-neutral measure, $X_{t}$ denote stock returns under risk-neutral measure. Stock price process $S_{t}$ under risk neutral measure can be expressed as $S_{t}=S_{0} \exp \left((r+\omega) t+X_{t}\right)$, from which $\omega$ satisfies $\mathrm{e}^{-\omega t}=\varphi_{X t}(-i)$. We suppose the returns process to follow tempered stable distribution and particularly pay attention to two kinds of tempered stable distributions: the classical tempered stable distribution and the normal tempered stable distribution. In Kim [34], characteristic functions of CTS and NTS distribution are calculated as:

$$
\begin{aligned}
& \varphi_{C T S_{X_{t}}}(u)=\exp \left(t C \Gamma(-\alpha)\left(\lambda_{-}-i u\right)^{\alpha}-\lambda_{-}^{\alpha}+\left(\lambda_{+}+i u\right)^{\alpha}-\lambda_{+}^{\alpha}\right), \\
& \varphi_{N T S_{X_{t}}}(u)=\exp \left(\frac{(1-\theta) t}{v \theta}\left(1-\left(1-\frac{\left(i u \mu-\frac{\sigma^{2} u^{2}}{2}\right) v}{1-\theta}\right)^{\theta}\right)\right),
\end{aligned}
$$

where $\Gamma$ is the gamma function, $C, \lambda_{-}, \lambda_{+}, \alpha$ denote parameters in classical tempered stable distribution model respectively, $\alpha \in(0,2)$ and $\alpha \neq 0, \lambda_{-}, \lambda_{+}, \alpha>0$. And $\mu, v, \sigma, \theta$ denote parameters in normal tempered stable distribution model respectively, from which $\mu \in \mathrm{R}, \theta \in(0,1), v, \sigma>0$.

Parameters $\lambda_{+}$and $\lambda_{-}$in classical tempered stable distribution grasp the decay rate of the positive and negative tails, with parameters $\lambda_{-}, \lambda_{+}, \alpha$ related to the tail weights. When $\lambda_{+}>\lambda_{-}\left(\lambda_{-}>\lambda_{+}\right)$, the returns distribution is left (right) skewed, otherwise, it exhibits symmetry in returns. Similarly, the parameter $\mu$ in normal tempered stable distribution determines the skewness of density function, $v$ controls the excessive kurtosis that exceeds the normal distribution, $\theta$ affects the shape of returns density function, meaning that it can capture economic information contents in financial markets. When specifying $\theta=1 / 2$, it leads to the special case of Normal Inverse Gaussian model, while $\theta$ approximating to zero, it generates the special case of Variance Gamma model. What can be seen from the above equation is that the higher kurtosis time series have the higher values of $\theta$ are.

As to the choice of random time change model for volatility clustering, because of the mean reverting property the square root CIR process is considered, which describes the volatility dynamic $y_{t}$ utilizing equation $d y_{t}=\kappa\left(\eta-y_{t}\right) d t+\lambda \sqrt{y_{t}} d W_{t}$, with parameters $\kappa, \eta, \lambda$ satisfying the condition $2 \kappa \eta>=\lambda^{2}$ to guarantee that the CIR process is strictly positive when changing measures. It serves as a measure of the time change rate by speeding up or slowing down the elapsed time according to the economic events. We define the economic stochastic time change variable $Y_{t}$ within time $[0, t]$ as $Y_{t}=\int_{0}^{t} y(\mathrm{~s}) d s$, then the characteristic function of $Y_{t}$ can be computed in the following:

$$
\varphi\left(u, t, y_{0}\right)=\frac{\exp \left(\frac{\kappa^{2} \eta t}{\lambda^{2}}\right)}{\cosh \left(\frac{\gamma t}{2}\right)+\frac{\kappa}{\gamma} \sinh \left(\frac{\gamma t}{2}\right)^{2 \kappa \eta / \lambda^{2}}} \exp \underbrace{y_{0} \frac{2 i u}{\kappa+\operatorname{co}\left(\frac{\gamma t}{2}\right) \gamma}},
$$

with $\gamma=\sqrt{\kappa^{2}-2 \lambda^{2} i u}$.

Then we introduce the tempered stable distribution into the CIR process to construct the time changed TS model process $Z_{t}$ with $Z_{t}=X_{Y_{t}}$ by subordinating the tempered stable distributions into the stochastic volatility process. When $X_{t}$ follows the NTS process, $Z_{t}$ is the time changed NTS process, similar to the time changed CTS process. It is known that the characteristic function of $Z_{t}$ can be calculated as follows:

$$
\varphi_{Z_{t}}(u, t)=\varphi_{Y_{t}}\left(-i \Psi_{X_{t}}(u), t\right),
$$

where $\Psi(u)$ denotes the corresponding characteristic exponent.

The pure jump infinite activity Lévy process driven stochastic volatility model consists of two parts: one part is a pure jump Lévy process that controls asset price dynamic evolution process, the other part is a stochastic volatility process that controls the strength of price fluctuations and jump frequency by adjusting the time change rate. The TSSV process is established through subordinating Lévy model to stochastic volatility process which can reflect the jumps behaviors and stochastic volatility of returns $\ln \left(S_{t} / S_{0}\right)$ simultaneously. It not only overcomes drawbacks of pure jump Lévy models that cannot reflect the diffusion process of underlying volatility, but also 
overcomes the defects of stochastic volatility models that cannot depict infinite jumping behaviors of returns. After combining these two features we can capture volatility smiles of options when fitting to market data effectively. Then the characteristic function formula of logarithmic returns for TSSV model is derived as follow:

$$
\begin{aligned}
e^{i u \ln \frac{S_{t}}{S_{0}}} & =\exp \left(i u r t+\frac{2 \Psi(u) Y_{0}\left(1-e^{-\Pi T}\right)}{2 \Pi-(\Pi-\kappa)\left(1-e^{-\Pi T}\right)}-\frac{k \eta}{\lambda^{2}}\left(2 \ln \left(1-\frac{\Pi-\kappa}{2 \Pi}\left(1-e^{-\Pi T}\right)\right)+(\Pi-\kappa) t\right)\right), \\
& =\exp (\text { iurt }+\tau(t, u))
\end{aligned}
$$

where $\Pi=\sqrt{\kappa^{2}-2 \Psi(u) \sigma^{2}}, \Psi(u)$ refers to the characteristic exponent of pure jump Lévy processes, $\kappa, \eta, \lambda$ are parameters of CIR stochastic volatility process.

Under the risk-neutral measure $Q$, the characteristic function of returns process is the product of characteristic function under real probability measure $P$ and mean correction factor $\exp ((r-q) t) / E\left[\exp (S(t)) \mid v_{0}\right]$, whose risk-neutral probability density function can be obtained by fast Fourier transform of characteristic functions. In Fig. 1 we show the effect of four parameters of CTS marginal distribution in stochastic volatility classical tempered stable model have on density function distribution of CTSSV model, and in Fig. 2 we show the influence of four parameters of NTS marginal distribution in stochastic volatility normal tempered stable model have on the density function distribution of NTSSV model. What can be seen from figure is that parameters $C$ and $\alpha$ have inverse relationship with the peak sharpness of CTSSV model density function, while $\lambda_{ \pm}$affect the overall position of density distribution in CTSSV model, $\theta$ and $v$ are oppositely correlated with the sharpness of the peak in NTSSV model's density function, $\mu$ influences the overall position of NTSSV density distribution, and $\sigma$ affects the shape of the density function.
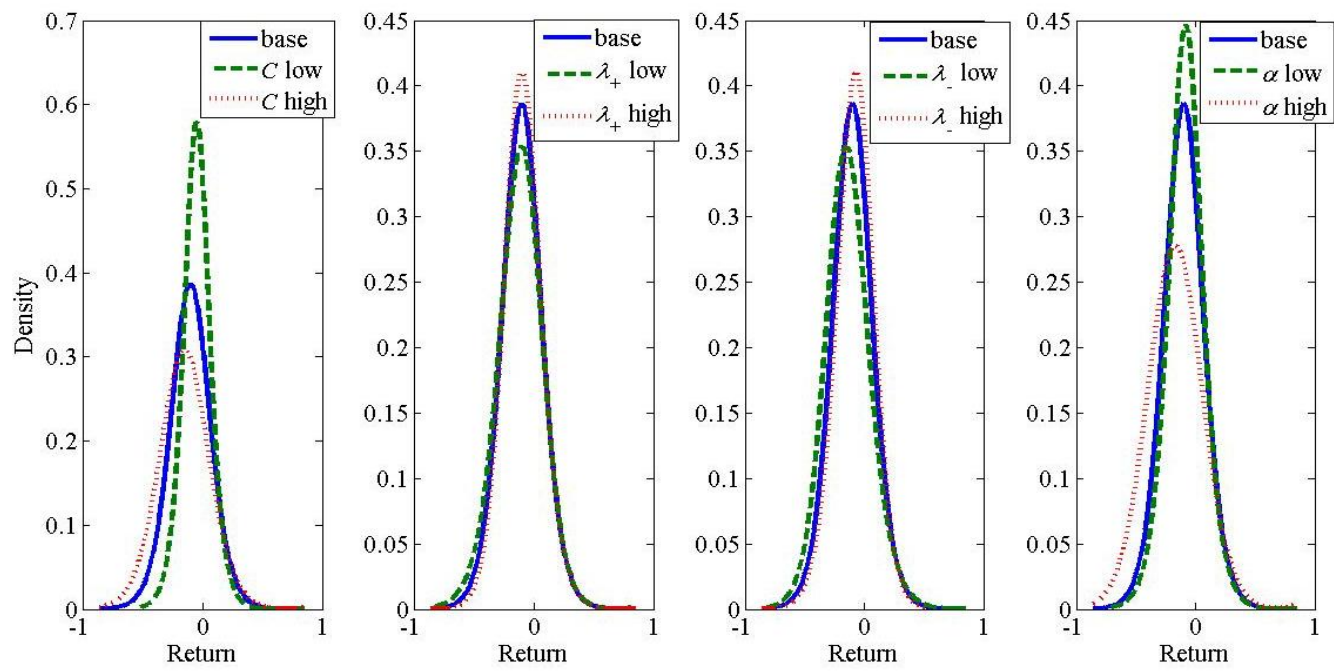

Fig.1. Risk-neutral probability density distribution of CTSSV model
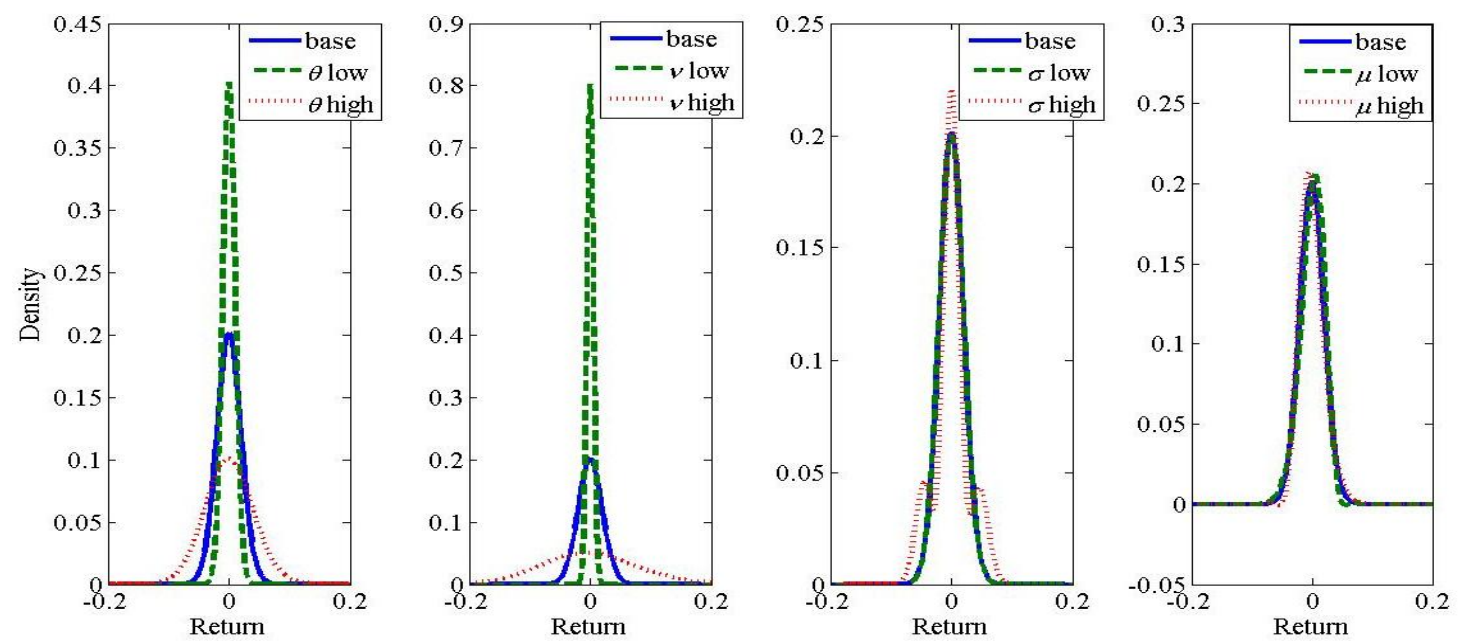

Fig.2. Risk-neutral probability density distribution of NTSSV model 


\section{Fourier-cosine method for TSSV American option valuation}

Because the stochastic volatility tempered stable processes exist analytical characteristic functions, Fourier based techniques can be efficiently applied to exotic option valuations. Fang [35] and Lord [25] respectively introduced the Fourier-cosine method and Fourier-convolution method for pricing American option in exponential Lévy models where a novel quadrature based method is utilized for options with early exercise feature, among them the former technique is illustrated more stable than the latter. It has been evidenced that employing Fourier-cosine method can achieve reasonable accuracy quite quickly, so that it is possible to calibrate a model to the prices of American options. American option can be exercised any time before maturity $T$, to which the repeated Richardson Extrapolation technique presented by Chang [36] can be used to price such option from Bermudan option quickly and accurately. The repeated Richardson extrapolation techniques are employed to enhance the computational efficiency and accuracy of American option. This method supposes that the American option $A_{\Delta}\left(t_{0}\right.$, $\left.S t_{0}\right)$ can be approximated from the price of Bermudan option $V_{\Delta}\left(t_{0}, S t_{0}\right)$ employing four point Richardson Extrapolation technique with respect to $\Delta_{k}=2^{-k}, k=n, n+1, n+2, n+3$ by $A_{\Delta}\left(t_{0}, \quad S t_{0}\right)=(1 / 21) \times\left[64 V_{\Delta(n+3)}\left(t_{0}\right.\right.$, $\left.\left.S t_{0}\right)-56 V_{\Delta(n+2)}\left(t_{0}, S t_{0}\right)+14 V_{\Delta(n+2)}\left(t_{0}, S t_{0}\right)-V_{\Delta}\left(t_{0}, S t_{0}\right)\right]$. Therefore, the American option valuation of stochastic volatility tempered stable model is transformed into the Bermudan option valuation of stochastic volatility tempered stable model, where we introduce the Fourier-cosine method that had been tested an efficient algorithm in evaluating pure jump Lévy processes into our constructed TSSV process scheme.

Let $v\left(x, t_{m}\right), c\left(x, t_{m}\right)$ and $g\left(x, t_{m}\right)$ respectively denote the option price, the continuation value and the corresponding payoff at time $t_{m}$ on grid points $x, \Delta_{t}=t_{m}-t_{m-1}, t_{0}<t_{1}<\ldots<t_{M}=T$, then the option pricing formula for $m=M, M-1, \ldots 2$ satisfies:

$$
\begin{aligned}
& c\left(x, t_{m}\right)=e^{-r \Delta_{t}} \int_{-\infty}^{+\infty} v\left(y, t_{m+1}\right) f(y \mid x) d y, \\
& v\left(x, t_{m}\right)=\max \left(g\left(x, t_{m+1}\right), c\left(x, t_{m+1}\right)\right) .
\end{aligned}
$$

The principal of Fourier-cosine method is due to that the cosine series coefficients of the probability density function $f$ are associated with their corresponding characteristic function, which can be computed through cosine series expansion on truncated range $(a, b)$ :

$$
f(y \mid x)=\sum_{k=0}^{N-1} A_{k}(x) \cos \left(k \pi \frac{y-a}{b-a}\right),
$$

where series coefficients $A_{k}(x)$ is expressed as $A_{k}(x)=\frac{2}{b-a} \int_{a}^{b} f(y \mid x) \cos \left(k \pi \frac{y-a}{b-a}\right) d y$.

By utilizing the relationship between $A_{k}(x)$ and characteristic function $\varphi(u)$ of the stochastic volatility tempered stable processes, it yields:

$$
A_{k}(x)=\frac{2}{b-a} \operatorname{Re}\left(e^{-i k \pi \frac{a}{b-a}} \int_{a}^{b} e^{i \frac{k \pi}{b-a} y} f(y \mid x) d y\right)=\frac{2}{b-a} \operatorname{Re}\left(e^{-i k \pi \frac{a}{b-a}} \varphi_{T S S V}\left(\frac{k \pi}{b-a}\right)\right) .
$$

Then the cosine formulation of option valuation for stochastic volatility tempered stable processes can be calculated as follows:

$$
c\left(x, t_{m+1}\right)=e^{-r \Delta t} \sum_{k=0}^{N-1} \operatorname{Re}\left(e^{-i k \pi \frac{x-a}{b-a}} \varphi_{T S S V}\left(\frac{k \pi}{b-a}\right)\right) V_{k}\left(t_{m+1}\right),
$$

where $V_{k}\left(t_{m+1}\right)$ denotes the $k$ th cosine series coefficients of option value $v\left(y, t_{m}\right)$, which consists of two ingredients. One integral $C\left(a, x^{*}, t_{m},\right)$ ranges from $a$ to the early exercise boundary $x^{*}$ at time $t_{m}$ is determined by Newton's method in root-finding algorithm, the other integral $G\left(a, x^{*}, t_{m},\right)$ ranges from early exercise boundary $x^{*}$ to $b$, which are calculated analytically in the following respectively.

$$
\begin{aligned}
& G_{k}\left(x_{1}, x_{2}, t_{m}\right)=\left\{\begin{array}{l}
\frac{2}{b-a} K\left(\chi_{k}\left(x_{1}, x_{2}\right)-\Psi_{k}\left(x_{1}, x_{2}\right)\right), \text { Call } \\
\frac{2}{b-a} K\left(\Psi_{k}\left(x_{1}, x_{2}\right)-\chi_{k}\left(x_{1}, x_{2}\right)\right), \text { Put }
\end{array},\right. \\
& C\left(x_{1}, x_{2}, t_{m}\right)=\frac{e^{-r \Delta t}}{\pi} \operatorname{Im}\left(\left(M_{c}+M_{s}\right) u\right),
\end{aligned}
$$

where $K$ is the strike range of option, Im denotes getting the imaginary section of the input argument, $u$ denotes the diagonal matrix with characteristic function contents $\varphi_{T S S V}(u)$ of stochastic volatility tempered stable processes, and the specific computation representation of the above equalities are given via employing $e^{i \alpha}=\cos \alpha+i \cdot \sin \alpha$ in $M_{c}$ and $M_{s}$. 


$$
\begin{aligned}
& \chi_{k}\left(x_{1}, x_{2}\right)=\frac{1}{1+\left(\frac{k \pi}{b-a}\right)^{2}}\left(\begin{array}{l}
\cos \left(k \pi \frac{x_{2}-a}{b-a}\right) e^{x_{2}}-\cos \left(k \pi \frac{x_{1}-a}{b-a}\right) e^{x_{1}}+\frac{k \pi}{b-a} \sin \left(k \pi \frac{x_{2}-a}{b-a}\right) e^{x_{2}} \\
-\frac{k \pi}{b-a} \sin \left(k \pi \frac{x_{1}-a}{b-a}\right) e^{x_{1}}
\end{array}\right), \\
& \Psi_{k}\left(x_{1}, x_{2}\right)=\left\{\begin{array}{l}
\left(\sin \left(k \pi \frac{x_{2}-a}{b-a}\right)-\sin \left(k \pi \frac{x_{1}-a}{b-a}\right)\right) \frac{b-a}{k \pi} k \neq 0, \\
d-c \quad k=0
\end{array}\right. \\
& M_{k, j}^{c}=\left\{\begin{array}{ll}
\frac{\left(x_{2}-x_{1}\right) \pi i}{b-a} & k=j \\
\frac{\exp \left(i(j+k) \frac{\left(x_{2}-a\right) \pi}{b-a}\right)-\exp \left(i(j+k) \frac{\left(x_{1}-a\right) \pi}{b-a}\right)}{j+k} & \text { else }
\end{array},\right. \\
& M_{k, j}^{s}=\left\{\begin{array}{ll}
\frac{\left(x_{2}-x_{1}\right) \pi i}{b-a} & k=j=0 \\
\frac{\exp \left(i(j-k) \frac{\left(x_{2}-a\right) \pi}{b-a}\right)-\exp \left(i(j-k) \frac{\left(x_{1}-a\right) \pi}{b-a}\right)}{j-k} & \text { else }
\end{array},\right.
\end{aligned}
$$

from which $j=0,1, \ldots N-1, i$ denotes the imaginary unit. And FFT algorithm can be efficiently applied to the matrices multiplication.

As to the truncation range $(a, b)$, Fang [35] proposed the choice formula for the integration range as follows:

$$
(a, b)=\left(\left(c_{1}+\ln \left(S_{0} / K\right)\right)-L \sqrt{c_{2}+\sqrt{c_{4}}},\left(c_{1}+\ln \left(S_{0} / K\right)\right)+L \sqrt{c_{2}+\sqrt{c_{4}}}\right),
$$

where the determination of $L$ relies on the tolerance level, $c_{1}, \ldots c_{4}$ represent the cumulants of the characteristic functions for TSSV models. The reason for inclusion of $c_{4}$ is that the probability density functions of the underlying assets possess sharp peaks and fat tails behaviors that can be adequately captured by it. With the Fourier-cosine method at hand, the calibration problem for American option under TSSV processes is now within reach.

\section{Improved PSO intelligent algorithm for model calibration}

Parameters estimation of stochastic volatility tempered stable Lévy processes models involve high dimensional integral variables, most of whose density functions have no analytical forms, thus making likelihood estimation method and moment estimation method computationally difficult. Besides, the calibration to actual market data constructs a nonlinear problem, making the common optimization algorithms no longer provide reliable results. The introduction of stochastic volatility brings more parameters that increase computation burden in calibration. As a consequence, the traditional optimization techniques may fail to obtain the optimal solution. However, the analytical existence of their characteristic functions makes intelligent optimization algorithm for effective calibration available.

Basic PSO algorithm searches the optimal solution through collaboration and information sharing between groups of individuals at each of the iteration. Evaluate the objective function of every particle, and determine the best location pbest each particle has passed at time $t$ as well as the group best location gbest, then updates particles' velocity and position iteratively. Because particles in PSO algorithm move towards the historically best position of their own and gather around the neighborhood of groups' best positions, they give rise to the formation of rapid convergence effect of particle populations which opt to fall into local minimum or converge prematurely. Moreover, the use of real options market data to estimate nonlinear optimization model parameter is also easy to fall into local minimum, leading derivatives pricing inaccurate. Therefore, it brings significance on the improvement of PSO algorithm.

In PSO it is assumed that each particle moves in an $n$-dimensional space, $X_{i}=\left(x_{i l}, x_{i 2}, \ldots, x_{i n}\right)$ refers to the current position of particle $i, V_{i}=\left(v_{i l}, v_{i 2}, \ldots, v_{i n}\right)$ denotes the current flight speed of particle $i, p_{i}=\left(\right.$ pbest $_{i l}$, pbest $_{i 2}, \ldots$, pbest $_{i n}$ ) is the individual best position of particle $i$. The particle dynamically adjusts the velocity and position according to the individual and group flying experience. What can be seen is that the flight speed of particles affects the global convergence of the algorithm. When the particle velocity is set too large, it ensures particles fly over global optimal solution area quickly, while approaching the optimal solution, due to the lack of effective control and constraints of flying particles, it is easy to overfly the optimal solution, turning to other areas which give local optimum. In order to estimate the TSSV model effectively, we develop the IPSO algorithm, that is, non-linear dynamic inertia weight PSO algorithm. Because of their speed and position of the particles depending on the parameter settings, the IPSO algorithm with improved global search ability is obtained by introduction of inertia 
weight $\omega$. Then the velocity and position of each particle are updated via pbest and gbest as follows.

$$
\begin{aligned}
& v_{i j}(t+1)=\omega v_{i j}(t)+c_{1} r_{1}\left(\text { pbest }_{i j}(t)-x_{i j}(t)\right)+c_{2} r_{2}\left(\text { gbest }_{j}(t)-x_{i j}(t)\right), \\
& x_{i j}(t+1)=x_{i j}(t)+v_{i j}(t+1)
\end{aligned},
$$

where $\omega$ refers to the inertia weight, both $c_{1}$ and $c_{2}$ denote the cognitive coefficients, $r_{1}$ and $r_{2}$ represent random numbers uniformly distributed between $(0,1), c_{1}$ can adjust the flight step of particles flying to their best position, $c_{2}$ can adjust particles flight steps to the population optimum, gbest $t_{j}(t)$ is the $j$ th dimensional components of the optimal position when the particle group evolved to generation $t$.

For the sake of balancing global search capability and local orientation ability of algorithm, when the target value of each particle tends to converge or approach to local optimization, inertia weight is increased, while the target values of each particle are scattered, the inertia weight is reduced. Meanwhile for particles that have higher objective functions values than the average, we assign smaller inertia weight factor so as to retain the particles. On the contrary, for particles with lower objective functions values than the average, they are assigned larger inertia weight factor, so that the particles move closer to a reasonable search area. Nonlinear dynamic inertia weights of particles change with the value of objective function automatically, whose formula is expressed in the following:

$$
\omega=\left\{\begin{array}{l}
\omega_{\text {min }}-\frac{\left(\omega_{\text {max }}-\omega_{\text {min }}\right) \times\left(f-f_{\text {min }}\right)}{f_{\text {avg }}-f_{\text {min }}} \quad f \leq f_{\text {avg }}, \\
\omega_{\text {max }} f>f_{\text {avg }}
\end{array}\right.
$$

where $f$ denotes the objective function value of particles, $f_{\text {avg }}$ and $f_{\min }$ denote the average objective value and the minima objective value of particles respectively.

The other problem with PSO algorithm is that it loses diversity in the searching process, signifying that the mutation operator can also increase swarm's diversity. Recently, Chen [37] proposed a new PSO algorithm that brought an aging leader to enhance the diversity. To increase diversity of swarms in PSO, the IPSO algorithm considers both the dynamic inertia weights as well as the aging leader to improve the searching efficiency with a high level of diversity. As we all know, particles with higher degree of diversity will achieve enhanced performance. According to [37], it employed an operator of lifespan control to adjust the leader's age, while we computing the leading power utilizing the improved ratio, which can be expressed as

$$
\text { Ratio }=\left|\frac{f\left(\text { gbest }_{i}\right)-f\left(\text { gbest }_{i-1}\right)}{f\left(\text { gbest }_{i-1}\right)}\right| .
$$

The lifespan of the gbest increases in accordance with its leading power that is reflected by the Ratio value above with the same operator control rule as Chen [37] in which updated scenarios of leaders are categorized into four cases. We iterate the particle's leader consisting with the previous, while differentiate form it due to randomly chosen particles which enables the learning object to be diversified.

IPSO algorithm is designed to get over the problem of premature convergence of traditional PSO algorithm without remarkably impairing the fast converging character of PSO algorithm. Theoretically, the proposed IPSO intelligent algorithm shares many advantages of the traditional PSO algorithm, but overcomes its drawbacks. Subsequently, it will be applied to our TSSV models for American option valuation calibration to test its effect in financial models.

\section{Empirical results}

\subsection{Descriptive statistics}

We empirically assess the performances of two different families of stochastic process models to evaluate the prices of American option. By utilizing the Fourier-cosine method and improved particle swarm intelligent optimization algorithm for stochastic volatility tempered stable model in American option valuation, the OEX American option data across a range of maturities in Bloomberg on Thursday 31 March 2016 is investigated. The SP 100 index value traded on that day is 915.75 . And the value of the risk-free rate of return is $0.59 \%$. We compare three classes of time varying infinite activity Lévy processes including stochastic volatility Normal Inverse Gaussian model, stochastic volatility classical tempered stable model and stochastic volatility normal tempered stable model to examine the modeling effect, and the normal tempered stable model exclusive of stochastic volatility diffusion component is also computed for comparison purpose. Before empirical research, we examine the returns characteristics of SP100 index from 2010.1.4 to 2015.12.1. It has been validated that SP100 index exhibits leptokurtic and fat tails properties with its skew value -0.1862 and kurtosis value 6.7023 . The negative skew value indicates that returns distribution is left skewed and the higher kurtosis value exceeding 3 than the value of normal distribution indicates its leptokurtosis property. The $Q Q$ plot of SP100 index returns in Fig.3 on the left graph confirms its asymmetry nature, and the returns sequences signify its volatility clustering nature and infinite 
jumps property.
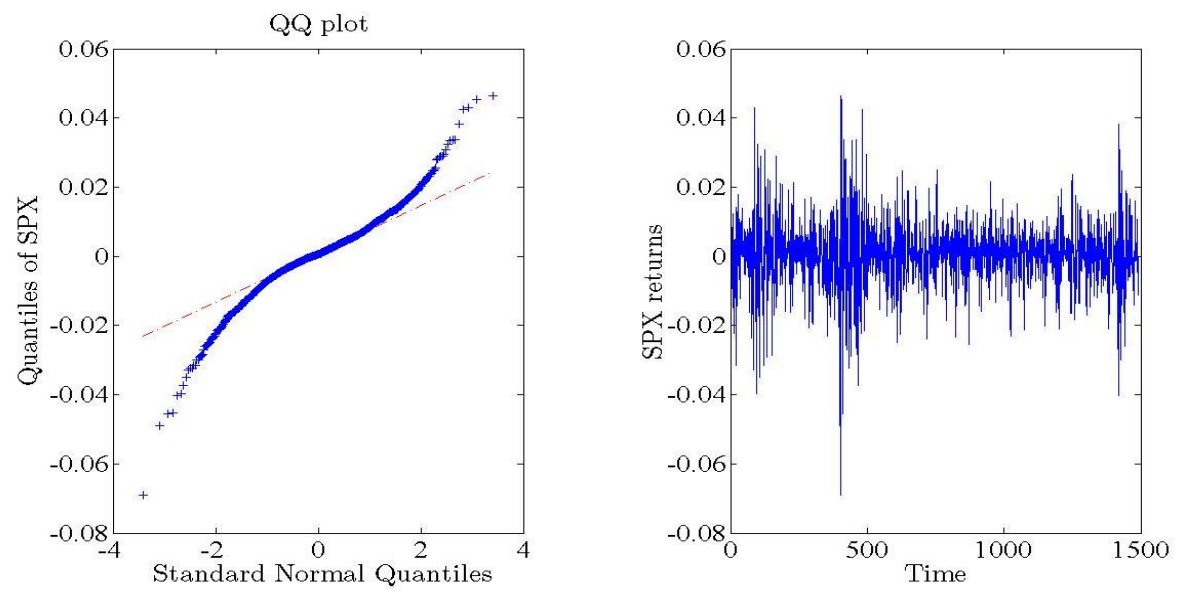

Fig.3. QQ plot and returns series of index

\subsection{Calibration results}

From a practical perspective, we minimize the objective function given by the root mean square error (RMSE) between the real market observables and the model generated prices. In addition, we give the definition of average absolute percentage error (ARPE):

$$
\begin{aligned}
& R M S E=\sqrt{\sum_{t_{i}} \frac{\left(O_{\text {market }}-O_{\text {model }}(\Theta)\right)^{2}}{N}}, \\
& A R P E=\frac{1}{O_{\text {average }}} \sum_{i=1}^{N} \frac{\left|O_{\text {market }}-O_{\text {model }}(\Theta)\right|}{N},
\end{aligned}
$$

where $t_{i}$ denotes different maturities, $O_{\text {model }}$ denotes the option prices obtained from established models, $\Theta$ denotes the parameters set, $O_{\text {market }}$ denotes the market observables of SP100 index option.

Since the minimization of the above equation with respect to the parameters set constitutes an ill-posed problem that neither has analytical solutions nor guarantee global optimum. Therefore, we use the IPSO intelligent algorithm to optimize it so as to achieve global optimality. During the optimization procedure, the NTSSV parameters $(\mu, v, \sigma, \theta, \kappa, \eta, \lambda)$ move into the region between $(-0.1,0,0.1,0,0.01,-5,-5)$ and $(0.1,20,0.1,1,10,10$, $5)$, the CTSSV parameters $\left(C, \lambda_{-}, \lambda_{+}, \alpha, \kappa, \eta, \lambda\right)$ move into the region between $(0.01,5,0.1,0.2,0.01,-5,-5)$ and $(2$, $30,20,1,10,10,5)$, the NIGCIR parameters $(\alpha, \beta, \delta, \kappa, \eta, \lambda)$ move into the region between $(0.01,-19,0.01,0.01$, $-5,-5)$ and $(20,19,10,10,10,5)$. Lower and upper bounds parameters have been introduced to restrict the search space to suitable solutions. The number of particles is 50 , cognitive coefficients $c_{1}=c_{2}=2$, inertia weights $\omega_{\max }=0.9$, $\omega_{\min }=0.6$. The corresponding model calibration results are displayed in Table 1, which will be further used for option pricing subsequently.

Table 1

Model calibration results

\begin{tabular}{cccccccc}
\hline Model & \multicolumn{7}{c}{ Parameter } \\
\hline \multirow{2}{*}{ NIGCIR } & $\alpha$ & $\beta$ & $\delta$ & $\kappa$ & $\eta$ & $\lambda$ & \\
& 17.3501 & -6.9182 & 5.6671 & 9.3751 & 0.0877 & 0.6122 & \\
CTSSV & $C$ & $\lambda$ & $\lambda_{+}$ & $\alpha$ & $\kappa$ & $\eta$ & $\lambda$ \\
& 5.2138 & 19.1139 & 24.0052 & 0.6173 & 7.4505 & 0.0824 & 0.8826 \\
NTSSV & $\mu$ & $v$ & $\sigma$ & $\theta$ & $\kappa$ & $\eta$ & $\lambda$ \\
& -0.0425 & 6.9716 & 0.0822 & 0.8347 & 7.9214 & 0.0866 & 0.9142 \\
NTS & $\mu$ & $v$ & $\sigma$ & $\theta$ & & & \\
& -0.0627 & 12.1754 & 0.0914 & 0.8214 & & & \\
\hline
\end{tabular}

The RMSE values of models in Table are respectively 4.5581, 3.4209, 2.5174, and 5.4470. From the estimations above it can be seen that the NTSSV model fits the data best, and seems the most appropriate model for financial stocks. Besides inheriting the properties in NTS model, the NTSSV model also possesses the superiority of permitting time varying variances in returns. The parameters $\theta$ that determine the distribution of density functions are similar with both values of 0.8 , whereas parameters $v$ that reflect the intricate structure in volatility in NTS with value of 12.1754 is much larger than that in NTSSV with value of 6.9716 , signifying that the NTS model has difficulty in accounting for uncertain volatility information of market future expectation. Both the values of $\mu$ in 
NTS and NTSSV models are below zero, indicating that the density distribution of SP100 index is left skewed. Noticeably, the NTS model exhibits competitive performance with NIGCIR model taking into consideration of its fewer parameters. The consequences of CTSSV model with $\lambda_{+}=24.0052>\lambda_{-}=19.1139$ and negative value of $\beta=$ -6.9182 in NIGCIR model also validates the returns distribution is left skewed. When it comes to the volatility diffusion components, the values of volatility reverting rate $\kappa$, variance of volatility $\eta$ in TSSV models are lower than that in NIGCIR model, this is mainly due to the strong power of tempered stable processes in capturing infinite activity properties in returns which decrease the persistence of the volatility diffusion.

In order to examine the newly established model, we calculate the implied volatility surface of our calibrated NTSSV option pricing models across different maturities with wide ranges of strikes, which are exhibited in Fig. 4. The results shows that the NTSSV model exhibits the volatility skew efficiently which can better reflect market behavior. Incorporating random time change into the tempered stable processes enables the models to accomplish the valuation of options more accurately.

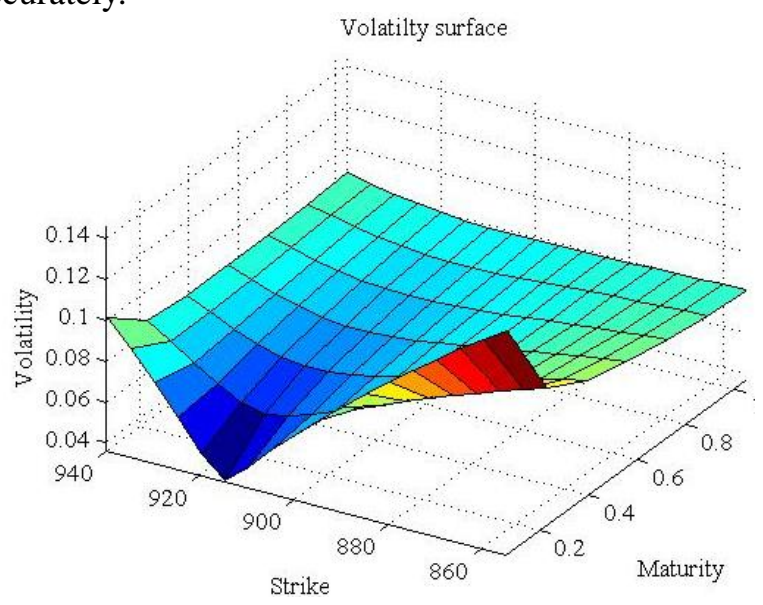

Fig. 4. The volatility surface of NTSSV model

By utilizing the IPSO algorithm, we obtain the optimal estimation consequences across a variety of models with the actual option prices. During the calibration procedure the maximum number of iteration generation is chosen 200 with 50 population size of particles. Fig. 5 displays the convergence conditions of intermediate objective functions applied to CTSSV model and NTSSV model, employing PSO and IPSO algorithms respectively. What can be seen from the figure is that it needs about 120 generations for CTSSV model and 110 generations for NTSSV model by PSO algorithm to reach a stable minimum value. However, it only takes roughly 90 generations in both models for IPSO to converge. The convergence performance of the PSO algorithm is relatively worse than that of the IPSO algorithm. It is the IPSO algorithm that clearly produces more efficient results.
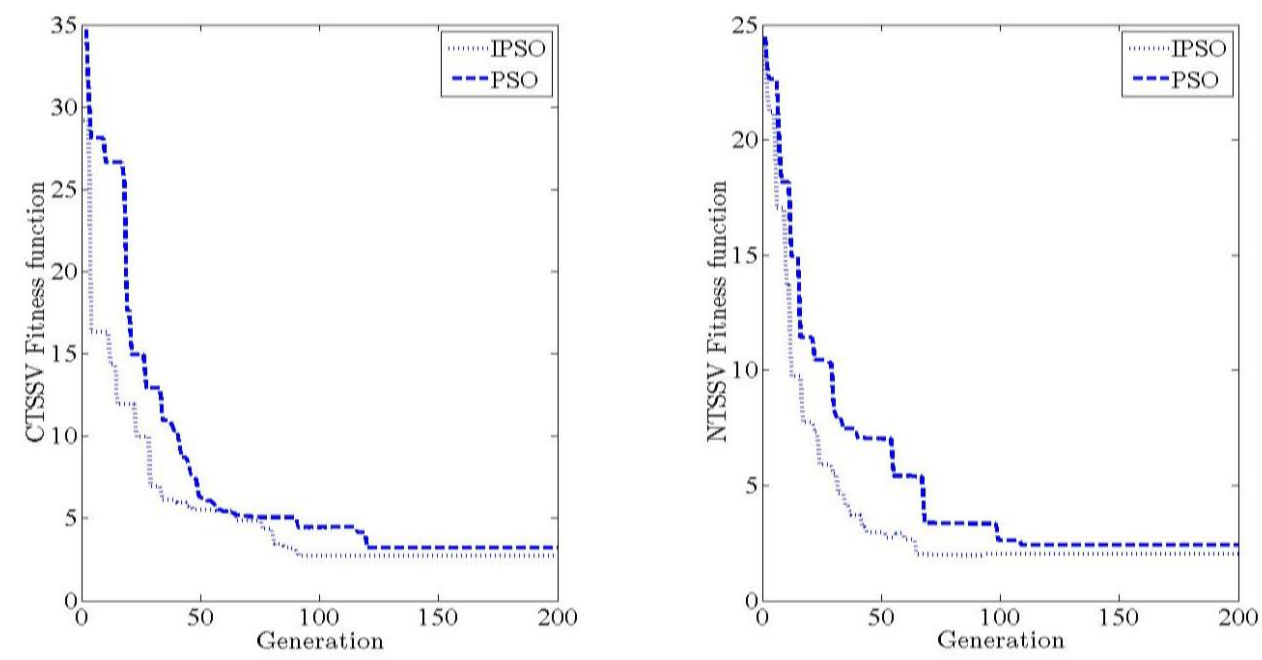

Fig. 5. Convergence of objective functions about IPSO and PSO algorithms

\subsection{Option pricing results}

Armed with perfect calibration results and powerful calculation tools, we will price OEX American put options on that day including maturities less than 200 days. We conduct empirical experiments on short term 
maturity ( $<40$ days), medium term maturity (40-90days) and long term maturity ( $>90$ days) options assuming the stock returns following the CTSSV stochastic process and NTSSV stochastic process as well as NTS stochastic process. Table 2 exhibits the RMSE and ARPE values produced from the IPSO algorithm calibrated parameters by maturity category and moneyness $(\mathrm{K} / \mathrm{S})$ classification.

Table 2

Pricing errors in moneyness and maturity categories

\begin{tabular}{|c|c|c|c|c|c|c|c|}
\hline \multirow{2}{*}{ Models } & \multirow{2}{*}{$\begin{array}{c}\text { Moneyness } \\
(\mathrm{K} / \mathrm{S})\end{array}$} & \multicolumn{2}{|c|}{ Short maturity } & \multicolumn{2}{|c|}{ Medium maturity } & \multicolumn{2}{|c|}{ Long maturity } \\
\hline & & RMSE & ARPE & RMSE & ARPE & RMSE & ARPE \\
\hline \multirow{3}{*}{ CTSSV } & ITM $(<0.96)$ & 3.5122 & 0.0338 & 3.4231 & 0.0285 & 3.5315 & 0.0345 \\
\hline & $\operatorname{ATM}(0.96-1.04)$ & 3.1897 & 0.0342 & 3.3305 & 0.0329 & 3.5037 & 0.0402 \\
\hline & OTM(>1.04) & 3.4358 & 0.0459 & 3.6203 & 0.0401 & 3.5122 & 0.0413 \\
\hline \multirow{4}{*}{ NTSSV } & $\operatorname{ITM}(<0.96)$ & 2.9806 & 0.0271 & 2.9246 & 0.0225 & 2.9588 & 0.0299 \\
\hline & $\operatorname{ATM}(0.96-1.04)$ & 2.0021 & 0.0195 & 2.1231 & 0.0201 & 2.2608 & 0.0217 \\
\hline & OTM(>1.04) & 2.2977 & 0.0262 & 2.3057 & 0.0278 & 2.2217 & 0.0233 \\
\hline & $\operatorname{ITM}(<0.96)$ & 4.3455 & 0.0659 & 5.6244 & 0.0794 & 6.4520 & 0.0973 \\
\hline \multirow{2}{*}{ NTS } & $\operatorname{ATM}(0.96-1.04)$ & 4.1216 & 0.0623 & 5.9026 & 0.0722 & 6.1184 & 0.0814 \\
\hline & OTM(>1.04) & 5.2133 & 0.0744 & 5.8906 & 0.0901 & 6.0052 & 0.0826 \\
\hline
\end{tabular}

It is remarkable that when coming to calibration to real market data, the time changed tempered stable models outperform pure jumps tempered stable model. The infinite activity pure jumps NTS model is expert at reflecting skewness for the whole maturities, while it lacks degree of freedom reflecting stochastic volatility of the term structure, which is captured by time varying Lévy models especially for long term smiles. It is illustrated that stochastic volatility models in combination with infinite activity Lévy jumps add explanatory power to improve the empirical fitting efficiency. The respective average RMSE errors for CTSSV and NTSSV are 3.451 and 2.4528, whereas 5.5192 for NTS models. Specifically, time changed infinite jumps models give significant smaller pricing errors for long maturity and medium maturity options compared to NTS models for ATM and OTM options.

Nevertheless, the pricing errors of all the models become smaller for ATM options. The average RMSE value of NTSSV model is $35.42 \%$ smaller than that of CTSSV model respectively for OTM options, whereas for ITM options, the average RMSE values of NTSSV models is $15.31 \%$ lower than that of CTSSV model, indicating that infinite activity NTS Lévy process displays preponderance in OTM option valuation. The results of CTSSV model and NTSSV model are not completely different, with the stochastic time clock dominating over the details of the tempered stable models. The numerical results in table also indicate that the ARPE of NTSSV model is smaller than other processes. The case of longer maturity shows that NTSSV models provide relatively better consequences. In summary, the proposed TSSV scheme obtains high quality option prices with relatively smaller errors for American call.

During the calculation procedure, we approximate the American option through increasing the number of exercise opportunities of Bermudan option applying Richardson extrapolation technique. Then, we choose the actual market data as reference values and examine the RMSE pricing errors of CTSSV model and NTSSV model with the exercise opportunities ranging from 10 to 200. It turns out that when the exercise opportunities increase, both the pricing errors of models using Fourier-cosine method decrease with a smooth and stable convergence, which confirm the effectiveness of cosine method. The following plot examination on RMSE pricing error against exercise opportunity also shows that NTSSV model outperforms its competitors.

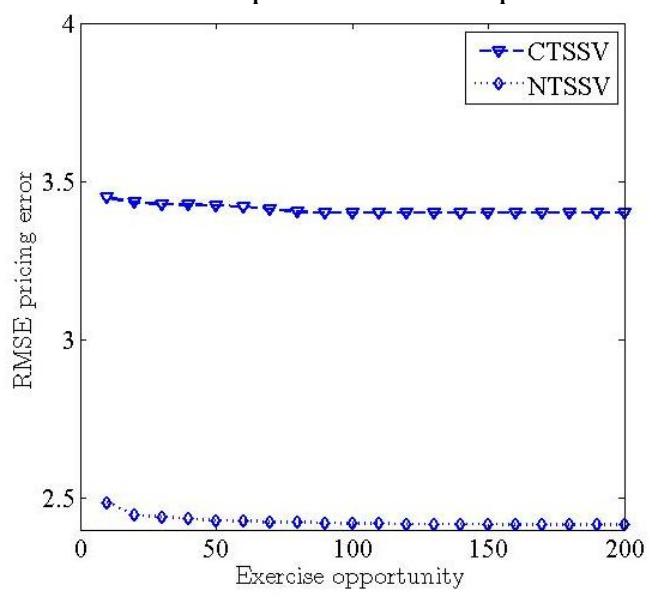

Fig.6. Calculation efficiency of CTSSV model and NTSSV model 


\section{Conclusion}

The Black-Scholes option pricing assumptions have been soundly rejected on empirical grounds since the leptokurtosis, asymmetry, clustering and heteroskedasticity effect observed in returns on financial assets. This paper employs the infinite activity properties of tempered stable processes and develops an extension of normal tempered stable model that incorporates Heston-type stochastic volatility to identify the distributional features in returns and volatility. The stochastic volatility normal tempered stable model accommodates stochastic time that can produce varying time and clustering volatilities in addition to permitting infinite jumps in underlying assets. The introduction of Fourier-cosine approach in combination with Richardson extrapolation technique makes the parameters calibration of American option feasible. Subsequently, we utilize the newly proposed improved particle swarm intelligent optimization algorithm to accelerate the calibration speed and efficiency.

Empirical studies show that the time changed tempered stable processes provide good fits for American option with different maturities, especially the NTSSV model, who delivers the smallest errors among them. It has so flexible structure that contains jump components as well as allowing the process affected by volatility dynamics. The inclusion of square root time changes into pure jumps infinite activity tempered stable distributions leads to significant improvements in American option valuation which demonstrate the superiority and applicability of our model scheme.

\section{Acknowledgments}

We sincerely thank our anonymous referee. We also would like to acknowledge the financial support from the National Science Foundation of China (No. 71171042, 71371044, 71571038).

\section{Appendix A. Tempered stable processes}

According to Lévy-Khintchine theorem, the characteristic function of infinite divisible distribution satisfies the following formula.

$$
E(\exp (i u X))=\exp \left(-i u O+\frac{\sigma^{2} u^{2}}{2}-\int_{-\infty}^{+\infty}\left(e^{i u x}-1-i u x_{|x| \leq 1}\right) \gamma d x\right) .
$$

where real number $o$ refers to the drift that determines the location of distribution, $\gamma$ denotes the Lévy measure, indicating the arrival rate of jumps.

Using characteristic functions, the cumulant $c_{n}$ of tempered stable processes can be calculated through

$$
c_{n}=\frac{1}{i^{n}} \frac{d^{n}}{d u^{n}} \log E\left(e^{i u X}\right) .
$$

As an effective tool in reflecting jump behaviors, Lévy measure can be obtained from the characteristic function by fast Fourier transform. The corresponding Lévy measure of CTS and NTS distributions are as follows:

$$
\begin{aligned}
& v_{C T S}(d x)=\frac{C e^{-\lambda_{+} x}}{x^{1+\alpha}} 1_{x>0}+\frac{C e^{-\lambda_{-}|x|}}{|x|^{1+\alpha}} 1_{x<0}, \\
& v_{N T S}(d x)=\sqrt{\frac{2}{\pi}}\left(\frac{v}{1-\theta}\right)^{\theta-1} \frac{e^{\frac{\mu x}{\sigma^{2}}}|x|^{-\theta-\frac{1}{2}}}{\sigma \Gamma(1-\theta)}\left(\mu^{2}-\frac{2(\theta-1) \sigma^{2}}{v}\right)^{\frac{\theta}{2}+\frac{1}{4}} K_{\theta+\frac{1}{2}}\left(\sqrt{\frac{x^{2}}{\sigma^{2}}\left(\frac{\mu^{2}}{\sigma^{2}}-\frac{2(\theta-1)}{v}\right)}\right),
\end{aligned}
$$

where $K$ denotes the modified Bessel function of the third kind. $v_{C T S}(\mathrm{R})=v_{N T S}(\mathrm{R})=\infty$, indicating that the CTS and NTS processes are pure jumps processes and possess infinite activity property.

\section{References}

[1] S. Mittnik, M.S. Paolella, S.T. Rachev, Diagnosing and treating the fat tails in financial return data, J. Empri. Finance 7 (2000), 389-416.

[2] W. L. Xiao, W. G. Zhang, W. J. Xua, X. L. Zhang, The valuation of equity warrants in a fractional Brownian environment, Physica A 391 (2012) 1742-1752.

[3] H. Kleinert, J. Korbel, Option pricing beyond Black-Scholes based on double-fractional diffusion, Physica A 449 (2016) $200-214$.

[4] S. G. Kou, A jump diffusion model for option pricing, Manag. Sci. 48 (2002) 1086-1101.

[5] C. Charles, L. J. Chen, C. D. Fuh, The pricing of risk and sentiment: A study of executive stock options, Financ. Manag. 42 (2013) 79-99.

[6] D. Madan, P. Carr, E. Chang, The variance gamma process and option pricing, Eur. Financ. Rev. 2 (1998) 79-105.

[7] O. E. Barndorff-Nielsen, Processes of normal inverse Gaussian type, Financ. Stoch. 2 (1997) 41-68.

[8] X. L. Gong, X. T. Zhuang, Option pricing for stochastic volatility model with infinite activity Lévy jumps, Physica A 455 (2016) $1-10$.

[9] J. Rosinski, Tempering stable processes, Stoch. Proc. Appl. 117 (2007) 677-707. 
[10] Y. Kim, S. Rachev, D. Chung, M Bianchi, The modified tempered stable distribution, GARCH models and option pricing. Prob. Math. Stat. 29 (2009) 91-117.

[11] Y. Kim, S. Rachev, M. Bianchi, F. J. Fabozzi, Tempered stable and tempered infinitely divisible GARCH models, J. Bank. Finance 34 (2010) 2096-2109.

[12] A. Constantinides, S.E. Savel'ev, Modelling price dynamics: A hybrid truncated Lévy flight GARCH approach, Physica A 392 (2013) 2072-2078

[13] J. Gajda, A. Wyłomańska, Tempered stable Lévy motion driven by stable subordinator, Physica A 392 (2013) 3168-3176.

[14] E. Barndorff-Nielsen, N. Shephard, Integrated OU processes and non-Gaussian OU-based stochastic volatility models, Scand. J. Stat. 30 (2003) 277-95.

[15] J. Huang, L. Wu, Specification analysis of option pricing models based on time-changed Lévy processes, J. Finance, 59 (2004) $1405-1440$

[16] P. Carr, L.R. Wu, Time-changed Lévy processes and option pricing, J. Financ. Econ. 71 (2004) 113-141.

[17] W. Schoutens, S. Symens, The pricing of exotic options by Monte-Carlo simulations in a Levy market with stochastic volatility, Int. J. Theor. Appl. Finance 6 (2003) 839-864.

[18] T.S. Zaevski, Y.S. Kim, F.J. Fabozzi, Option pricing under stochastic volatility and tempered stable Lévy jumps, Int. Rev. Financ. Anal. 31 (2014) 101-108

[19] A. Yamazaki, Pricing average options under time-changed Lévy processes, Rev. Deriv. Res. 17 (2014) 79-111.

[20] A. Itkin, P. Carr, Pricing swaps and options on quadratic variation under stochastic time change models-discrete observations case, Rev. Deriv. Res. 13 (2010) 141-176.

[21] P. Carr, R. Lee, L. Wu, Variance swaps on time-changed Lévy processes, Finance Stoch. 16 (2012) 335-355.

[22] M. L. Bianchi, F. J. Fabozzi, Investigating the performance of non-Gaussian stochastic intensity models in the calibration of credit default swap spreads, Comput. Econ. 46 (2015)243-273.

[23] Y. S. Kim, J. Lee, S. Mittnik, J. Park. Quanto option pricing in the presence of fat tails and asymmetric dependence, J. Econometrics 187 (2015) 512-520.

[24] H. Y. Wong, P.Q. Guan, An FFT-network for Lévy option pricing, J. Bank. Finance 35 (2011) 988-999.

[25] R. Lord, F. Fang, F. Bervoets, C. W. Oosterlee, A fast and accurate FFT-based method for pricing early-exercise options under Lévy processes, SIAM J. Sci. Comput 30 (2008) 1678-1705.

[26] J. Li, C. Favero, F. Ortu, A spectral estimation of tempered stable stochastic volatility models and option pricing, Comput. Stati. Data Anal. 56 (2012) 3645-3658.

[27] J. Li, Sequential Bayesian analysis of time-changed infinite activity derivatives pricing models, J. Bus. Econ. Statist 29 (2009) $468-480$.

[28] J. Kennedy, R. C. Eberhart, Particle swarm optimization, Proceedings of IEEE International Conference on Neural Networks, New Jersey, 1995, pp. 1942-1948.

[29] T. Krink, S. Paterlini, Multiobjective optimization using differential evolution for real world portfolio optimization, Comput Manag. Sci. 8 (2011) 157-179.

[30] B. Fastrich, P. Winker, Combining forecasts with missing data: making use of portfolio theory, Comput. Econ. 44 (2014) $127-152$.

[31] S. Yang, J. Lee, Multi-basin particle swarm intelligence method for optimal calibration of parametric Levy models, Expert Syst. Appl. 39 (2012) 482-493.

[32] Q. Sun, W. D. Xu, Pricing foreign equity option with stochastic volatility, Physica A 437 (2015) 89-100.

[33] P. Carr, H. Geman, D. Madan, M. Yor, Stochastic volatility for Lévy processes, Math. Finance 13 (2003) 345-382.

[34] Y. Kim, S. Rachev, M. Bianchi, F. J. Fabozzi, Financial market models with Lévy processes and time varying volatility, J. Banking. Finance, 32 (2008) 1363-1378.

[35] F. Fang, C. W. Osterlee, Pricing early-exercise and discrete barrier options by Fourier-cosine expansions, Numer. Math. 114 (2009) 27-62.

[36] C. C. Chang, S. L. Chung, R. C. Stapleton, Richardson extrapolation techniques for the pricing of American style options, J. Futures Markets 27 (2007) 791-817.

[37] W. N. Chen, J. Zhang, Y. Lin, N. Chen, Z. H. Zhan, H. S. H. Chung, Particle swarm optimization with an aging leader and challengers, IEEE Tran. Evol. Comput. 17 (2013) 241-258. 